\title{
FORMAL REDUCTION OF LINEAR DIFFERENCE SYSTEMS
}

\author{
Guoting Chen and Abdelmajid Fahim
}

The purpose of this paper is to give a reduction procedure for the construction of a Turrittin's canonical form associated with an invertible linear difference system. The nilpotent case is treated by methods of deformation of orbits under the adjoint representation of $\mathrm{GL}(n, \mathbf{C})$. We prove also a statement on uniqueness.

\section{Introduction and notations.}

Since Turrittin [8], the formal reduction of difference systems or of difference equations has been studied in many ways [4], [5], [7]. The different methods lead to the result of Turrittin either in forms of classification or in forms of formal solutions. To construct a canonical form for a given invertible linear difference system with nilpotent leading matrix we proceed by using the theory of orbits under the adjoint action of $\operatorname{GL}(n, \mathbf{C})$ on $\operatorname{gl}(n, \mathbf{C})$ [1]. Though the reduction procedure of both the differential and difference systems is similar, we encounter some phenomena which do not appear in the differential case.

We shall use the following notations.

- $K=\mathbf{C}((1 / x))$ is the field of formal power series with coefficients in $\mathbf{C}$. $\phi$ is the $\mathbf{C}$-automorphism of $K$ defined by $\phi(x)=x+1$.

- For $q \in \mathbf{N}^{*}, x^{1 / q}$ is a fixed root of $y^{q}=x, \mathcal{O}_{q}=\mathbf{C}\left[\left[\frac{1}{x^{1 / q}}\right]\right], K_{q}=$ $\mathbf{C}\left(\left(\frac{1}{x^{1 / q}}\right)\right), \bar{K}=\bigcup_{q \in \mathbf{N}^{*}} K_{q}$ is the field of formal Puisieux power series over $\mathbf{C}$.

- $\phi$ can be extended to $\bar{K}$ by $\phi\left(x^{1 / q}\right)=x^{1 / q}\left(1+\frac{1}{x}\right)^{1 / q}$.

- For $M \in \operatorname{gl}(n, \mathbf{C}), q \in N^{*}, \Sigma(M)$ denotes the set of eigenvalues of $M$ and

$$
\mu_{q}(M)=\max \{j \in \mathbf{N} \mid \exists \lambda, \mu \in \Sigma(M), \lambda-\mu=j / q\} .
$$

- For $A \in \operatorname{gl}\left(n, K_{q}\right), A \neq 0$,

$$
\operatorname{ord}(A)=\max \left\{\frac{r}{q} \mid r \in \mathbf{Z}, A \in x^{-r / q} \operatorname{gl}\left(n, \mathcal{O}_{q}\right)\right\} ;
$$

$\operatorname{ord}(0)=+\infty$ 
We consider systems of linear difference equations of the form

$$
\phi(u)=A u
$$

where $A \in \operatorname{GL}\left(n, K_{q}\right), q \in \mathbf{N}^{*}$. One can write $A=\sum_{m=0}^{\infty} \frac{A_{r+m}}{x^{(r+m) / q}}$. The matrix $A$ or its associate system is said of level 0 if

$$
A=I+\sum_{m=q}^{\infty} \frac{A_{m}}{x^{m / q}}
$$

of level $\leq 1$ if

$$
A=I+\sum_{m=0}^{\infty} \frac{A_{r+m}}{x^{(r+m) / q}}, r \in \mathbf{N}^{*}, 1 \leq r<q, A_{r} \neq 0,
$$

where $I$ denotes the $n \times n$ identity matrix. Let $T \in \mathrm{GL}\left(n, K_{q}\right)$. The change $\tilde{u}=T u$ transforms the system (1) to

$$
\phi(\tilde{u})=\tilde{A} \tilde{u}
$$

where

$$
\tilde{A}=T[A] \stackrel{\text { def }}{=} \phi(T) A T^{-1} .
$$

We shall say that the matrices $A, \tilde{A}$ (or the corresponding difference systems) are equivalent (under $\operatorname{GL}\left(n, K_{q}\right)$ ).

Definition 1. Let $p \in \mathbf{N}^{*}$. We shall say that $B \in \operatorname{GL}\left(n, K_{p}\right)$ is in canonical form if $B=\frac{1}{x^{r / p}} \bigoplus_{i=1}^{s} \frac{B_{i}}{x^{\ell_{i}}}$ with

- $r \in \mathbf{Z}, \ell_{i} \in \frac{1}{p} \mathbf{N}, \ell_{1}<\ell_{2}<\cdots<\ell_{s}$,

- $B_{i} \in \mathrm{GL}\left(n^{(i)}, \mathcal{O}_{p}\right), n^{(i)} \in \mathbf{N}^{*}, \sum_{i} n^{(i)}=n, B_{i}=\bigoplus_{\alpha=1}^{t_{i}} \lambda_{\alpha}^{(i)}\left(B_{\alpha}^{(i)}+\frac{C_{\alpha}^{(i)}}{x}\right)$,

$$
B_{\alpha}^{(i)}=I_{\alpha}^{(i)}+\frac{D_{\alpha, 1}^{(i)}}{x^{r_{\alpha, 1}^{(i)}}}+\cdots+\frac{D_{\alpha, j_{\alpha}^{(i)}}^{(i)}}{x^{r^{(i)}(i)}}
$$

where

$\lambda_{\alpha}^{(i)} \in \mathbf{C}^{*}, \lambda_{\alpha}^{(i)} \neq \lambda_{\beta}^{(i)}$ for $\alpha \neq \beta$,

$I_{\alpha}^{(i)}$ is the $n_{\alpha}^{(i)} \times n_{\alpha}^{(i)}$ identity matrix, $n_{\alpha}^{(i)} \in \mathbf{N}^{*}, \sum_{\alpha=1}^{t_{i}} n_{\alpha}^{(i)}=n^{(i)}$,

$r_{\alpha, j}^{(i)} \in \frac{1}{p} \mathbf{N}^{*}, r_{\alpha, 1}^{(i)}<r_{\alpha, 2}^{(i)}<\cdots<r_{\alpha, j_{\alpha}^{(i)}}^{(i)}<1, D_{\alpha, j}^{(i)} \in \operatorname{gl}\left(n_{\alpha}^{(i)}, \mathbf{C}\right)(1 \leq$ $\left.j \leq j_{\alpha}^{(i)}\right)$ are nonzero diagonal matrices,

$C_{\alpha}^{(i)} \in \operatorname{gl}\left(n_{\alpha}^{(i)}, \mathbf{C}\right)$ commutes with the $D_{\alpha, j}^{(i)}\left(1 \leq j \leq j_{\alpha}^{(i)}\right)$. 
We make the convention that for $j_{\alpha}^{(i)}=0, B_{\alpha}^{(i)}=I_{\alpha}^{(i)}$.

One may write a canonical form in some other equivalent forms. We have chosen these conditions to ensure uniqueness (Theorem 2). A canonical form for a difference system (or a matrix) of level $\leq 1$ is in the form

$$
I+\frac{D_{1}}{x^{r_{1}}}+\cdots+\frac{D_{k}}{x^{r_{k}}}+\frac{C}{x}
$$

where the $D_{j}(1 \leq j \leq k)$ are nonzerro diagonal matrices, $0<r_{1}<\cdots<r_{k}$ are rational numbers and the matrix $C$ commutes with the matrices $D_{j}(1 \leq$ $j \leq k$ ). This special canonical form is the same as in the differential case [1]. But for general difference systems the canonical from is more complicated.

We can state the result of Turrittin as follows:

Theorem 1. Let $q \in \boldsymbol{N}^{*}$ and $A \in \operatorname{GL}\left(n, K_{q}\right)$. Then there exists $p \in q \boldsymbol{N}^{*}$ such that $A$ is equivalent under $\mathrm{GL}\left(n, K_{p}\right)$ to a matrix in canonical form. If $A$ is of level $\leq 1$ then it is equivalent to a matrix in the canonical form (5).

We will give an effective method which proves the theorem in several steps. We treat at first the case of matrices of level 0 in Section 2 and of level $\leq 1$ in Section 5. We then consider the general case in Section 6. In Section 7 we establish a statement on uniqueness (Theorem 2) of the canonical form. The procedure is analogous to that used for differential systems [1] in the case of level $\leq 1$. There is a different phenomena which is unique to difference systems. More precisely, for a complete reduction of a difference system we need to do two reductions, one to reduce general difference systems to the case of level $\leq 1$ and another one to reduce a difference system of level $\leq 1$.

\section{Reduction of systems of level 0 .}

We begin by the reduction of systems of level 0 .

Proposition 1. Let $q \in \boldsymbol{N}^{*}$ and $A \in \mathrm{GL}\left(n, K_{q}\right)$ be of level 0 . Then there exists $T \in \operatorname{GL}\left(n, K_{q}\right)$ such that $T[A]=I+\frac{C}{x}$ with $C \in \operatorname{gl}(n, C)$ and $\mu_{q}(C)=$ 0 .

Proof. Write $A=I+\sum_{m=q}^{\infty} \frac{A_{m}}{x^{m / q}}$. We distinguish two cases:

(i) $\mu_{q}\left(A_{q}\right)=0$; then one can verify by direct computation the existence of $T \in \operatorname{GL}\left(n, \mathcal{O}_{q}\right)$ such that $T[A]=I+\frac{A_{q}}{x}$. 
(ii) $\mu_{q}\left(A_{q}\right)>0$. Let $\Sigma\left(A_{q}\right)=\left\{\lambda_{1}, \lambda_{2}, \ldots, \lambda_{p}\right\}$ with $\lambda_{1}-\lambda_{2}=\mu_{q}\left(A_{q}\right)$. Let $n^{\prime}$ be the multiplicity of $\lambda_{1}$ and $n^{\prime \prime}=n-n^{\prime}$. After a change of basis one can assume that

$$
A_{q}=\left(\begin{array}{cc}
A_{q}^{\prime} & 0 \\
0 & A_{q}^{\prime \prime}
\end{array}\right)
$$

where $A_{q}^{\prime} \in \operatorname{gl}\left(n^{\prime}, \mathbf{C}\right), A_{q}^{\prime \prime} \in \operatorname{gl}\left(n^{\prime \prime}, \mathbf{C}\right)$ with $\Sigma\left(A_{q}^{\prime}\right)=\left\{\lambda_{1}\right\}$.

Define

$$
\tilde{T}=\left(\begin{array}{cc}
x^{-1 / q} I_{n^{\prime}} & 0 \\
0 & I_{n^{\prime \prime}}
\end{array}\right) .
$$

We have $\tilde{T}[A]=I+\frac{\tilde{A}_{q}}{x}+\cdots$ where

$$
\tilde{A}_{q}=\left(\begin{array}{cc}
A_{q}^{\prime}-\frac{1}{q} I_{n^{\prime}} & 0 \\
* & A_{q}^{\prime \prime}
\end{array}\right) .
$$

Hence $\Sigma\left(\tilde{A}_{q}\right)=\left\{\lambda_{1}-\frac{1}{q}, \lambda_{2}, \ldots, \lambda_{p}\right\}, \mu_{q}\left(\tilde{A}_{q}\right)=\mu_{q}\left(A_{q}\right)-1$.

By repeating the same procedure a finite number of times, the former case occurs.

\section{A splitting lemma.}

Let $\mathcal{G}=\operatorname{gl}(n, \mathbf{C})$. For $M \in \mathcal{G}, \mathcal{G}_{M}$ and $[\mathcal{G}, M]$ denote respectively the kernel and the image of the adjoint homomorphism ad $(M)$. The following lemma (and its proof) is the difference analogue of the differential case ([1], Proposition 4.3, p. 30).

Lemma 1. Let $A \in \mathrm{GL}\left(n, K_{q}\right)$ be in one of the following forms,

$$
\text { (I) } \quad I+\sum_{m=0}^{\infty} \frac{A_{r+m}}{x^{(r+m) / q}}, \quad 1 \leq r<q
$$

or

$$
\text { (II) } \quad x^{-r / q} \sum_{m=0}^{\infty} \frac{A_{r+m}}{x^{m / q}}, \quad r \in Z ;
$$

where $A_{r+m} \in \mathcal{G}, A_{r} \neq 0$. Let $\mathcal{L} \subset \mathcal{G}$ be a linear subspace such that

$$
\mathcal{G}=\mathcal{L}+\left[\mathcal{G}, A_{r}\right]
$$

Then there exist sequences $\left(T_{m}\right)_{m \geq 1}$ in $\mathcal{G},\left(A_{r+m}^{\prime}\right)_{m \geq 0}$ in $\mathcal{L}$ such that

$$
T[A]=I+\sum_{m=0}^{\infty} \frac{A_{r+m}^{\prime}}{x^{(r+m) / q}}, \quad \text { in the case }(I)
$$


or

$$
T[A]=x^{-r / q} \sum_{m=0}^{\infty} \frac{A_{r+m}^{\prime}}{x^{m / q}}, \quad \text { in the case }(I I)
$$

where

$$
T=\prod_{m=1}^{\infty}\left(I+\frac{T_{m}}{x^{m / q}}\right)
$$

Moreover $A_{r+m}^{\prime}$ only depends on $A_{r}, A_{r+1}, \ldots, A_{r+m}$.

Proof. We prove the lemma for the case (II), the reasoning being the same for the other one. Let $A^{(0)}=A, T^{(0)}=I$. For $j \in \mathbf{N}^{*}$ we will define

$$
A^{(j)}=x^{-r / q}\left(A_{r}^{(j)}+\frac{A_{r+1}^{(j)}}{x^{1 / q}}+\cdots\right) \in \mathrm{GL}\left(n, K_{q}\right)
$$

with $A_{r}^{(j)}=A_{r}$ and

$$
T^{(j)}=I+\frac{T_{j}}{x^{j / q}}, \quad T_{j} \in \mathcal{G}
$$

such that

(1) $A_{r+p}^{(j)}=A_{r+p}^{(j-1)}, 0 \leq p \leq j-1$,

(2) $A_{r+j}^{(j)} \in \mathcal{L}$

(3) $A^{(j)}=T^{(j)}\left[A^{(j-1)}\right]$.

For $j=1$, we have

$$
\left(I+\frac{T_{1}}{(x+1)^{1 / q}}\right)\left(A_{r}+\frac{A_{r+1}}{x^{1 / q}}+\cdots\right)=\left(A_{r}^{(1)}+\frac{A_{r+1}^{(1)}}{x^{1 / q}}+\cdots\right)\left(I+\frac{T_{1}}{x^{1 / q}}\right) .
$$

Then

$$
A_{r}=A_{r}^{(1)}, \quad A_{r+1}=A_{r+1}^{(1)}+\left[A_{r}, T_{1}\right] .
$$

The assumption on $\mathcal{L}$ implies the existence of $T_{1}$ in $\mathcal{G}$ and $A_{r+1}^{(1)}$ in $\mathcal{L}$ such that the last equation is fulfilled.

By induction on $j$, one chooses $T_{j+1}$ and $A_{r+j+1}^{(j+1)}$ such that

$$
A_{r+j}^{(j)}=A_{r+j+1}^{(j+1)}+\left[A_{r}, T_{j+1}\right] .
$$

We have

$$
\begin{aligned}
A^{(j)}=T^{(j)} T^{(j-1)} \cdots & T^{(1)}[A] \\
& =x^{-r / q}\left(A_{r}+\frac{A_{r+1}^{(1)}}{x^{1 / q}}+\cdots+\frac{A_{r+j}^{(j)}}{x^{j / q}}+O\left(\frac{1}{x^{(j+1) / q}}\right)\right) .
\end{aligned}
$$


Define $T=\prod_{m=1}^{\infty}\left(I+\frac{T_{m}}{x^{m / q}}\right)$ and $A^{\prime}=T[A]$. Then

$$
A^{\prime}-A^{(j)} \in \operatorname{gl}\left(n, x^{-(j+1+r) / q} \mathcal{O}_{q}\right), \quad \text { i.e. } \quad A^{\prime}=\lim _{j \rightarrow \infty} A^{(j)} .
$$

$A^{\prime}$ has the desired properties.

Corollary 1 (Splitting lemma). Let notations be as above. Let $\Sigma$ be the set of eigenvalues of $A_{r}, P_{\lambda}$ be the matrix of the projection of $C^{n}$ on the eigenspace corresponding to $\lambda$ in $\Sigma$. Let $S$ be the semisimple part of $A_{r}$. Choose $\mathcal{L}=\mathcal{G}_{S}$. Then $A_{r+m}^{\prime}$ commutes with $P_{\lambda}$ for $m \in N$; moreover

$$
T[A]=\bigoplus_{\lambda \in \Sigma} A_{\lambda}^{\prime}
$$

where

$$
\begin{aligned}
& A_{\lambda}^{\prime}=I+\sum_{m=0}^{\infty} \frac{P_{\lambda} A_{r+m}^{\prime}}{x^{(r+m) / q}} \quad \text { in the case }(I), \\
& A_{\lambda}^{\prime}=x^{-r / q} \sum_{m=0}^{\infty} \frac{P_{\lambda} A_{r+m}^{\prime}}{x^{m / q}} \quad \text { in the case }(I I) .
\end{aligned}
$$

Remark. The case (I) of the above lemma and corollary corresponds to a difference system of level $\leq 1$ which will be used in the Section 5 and the case (II) for a general system in the Section 6.

\section{Standard triples.}

We present now some results which will be used in the next sections.

For $M \in \mathcal{G}, d(M)$ is the dimension of the $\mathrm{GL}(n, \mathbf{C})$-orbit of $M$ with respect to the adjoint representation of $\mathcal{G}$.

Proposition 2 ([6], [1]). Let $Y$ be a nonzero nilpotent in $\mathcal{G}$; then we can find $H, X \in \operatorname{sl}(n, C)$ such that

$$
[H, X]=2 X, \quad[H, Y]=-2 Y, \quad[X, Y]=H .
$$

$(Y, H, X)$ is called a standard triple.

Proposition 3 ([1]). Let $Y$ be a nilpotent and $(Y, H, X)$ a standard triple. Let $Z \in \mathcal{G}_{X}, Z \neq 0$. Suppose that $Y+Z$ is nilpotent. Then $d(Y+Z)>d(Y)$. 
For a standard triple $(Y, H, X)$, we have $\mathcal{G}=\mathcal{G}_{X} \oplus[\mathcal{G}, Y]$. Moreover there exists a basis $\left\{Z_{1}, \ldots, Z_{\ell}\right\}$ of $\mathcal{G}_{X}$ such that $Z_{1}=I, Z_{j} \in \operatorname{sl}(n, \mathbf{C})$ for $j \geq 2$ (see [1], p. 15) and

$$
\left[H, Z_{j}\right]=\lambda_{j} Z_{j}, \quad \lambda_{j} \in \mathbf{N}, 1 \leq j \leq \ell .
$$

In particular $\lambda_{1}=0$. Define $\Lambda=\sup _{1 \leq j \leq \ell}\left(\frac{\lambda_{j}}{2}+1\right)$, then $1 \leq \Lambda \leq n$.

$\left\{Z_{1}, \ldots, Z_{\ell}\right\}$ can be extended to a basis $\left\{Z_{1}, \ldots, Z_{\ell}, Z_{\ell+1}, \ldots, Z_{n^{2}}\right\}$ of $\mathcal{G}$ with the following properties:

$$
\text { For all } j>\ell, \quad\left[H, Z_{j}\right]=\lambda_{j} Z_{j}, \quad \lambda_{j} \in \mathbf{Z}, \quad\left|\lambda_{j}\right| \leq \max _{1 \leq i \leq \ell} \lambda_{i} .
$$

If $M \in \operatorname{gl}(n, \mathbf{C})$ is such that $[H, M]=c M$ for some $c \in \mathbf{Z}$ then

$$
x^{m H} M x^{-m H}=x^{c m} M, \quad \text { for } p \in \mathbf{N}^{*}, m \in(1 / p) \mathbf{Z} .
$$

One has in particular

$$
x^{m H} Y x^{-m H}=x^{-2 m} Y ; \quad x^{m H} Z_{j} x^{-m H}=x^{\lambda_{j} m} Z_{j} .
$$

\section{Systems of level $\leq 1$.}

\subsection{The nilpotent case.}

Let

$$
A=I+\sum_{m=0}^{\infty} \frac{A_{r+m}}{x^{(r+m) / q}} \in \mathrm{GL}\left(n, K_{q}\right), 1 \leq r<q
$$

with $A_{r} \neq 0$. Assume that $Y=A_{r}$ is nilpotent. Let $(Y, H, X)$ be a standard triple. In the sequel we shall describe the first steps in order to reduce $A$.

\section{Lemma 2.}

(i) There exists $T \in \mathrm{GL}\left(n, \boldsymbol{C}\left[\frac{1}{x^{1 / q}}\right]\right)$ such that

$$
T[A]=I+\sum_{m=0}^{\infty} \frac{A_{r+m}^{\prime}}{x^{(r+m) / q}}
$$

with $A_{r}^{\prime}=A_{r}, A_{r+m}^{\prime} \in \mathcal{G}_{X}$ for $1 \leq m<\Lambda(q-r)$. Furthermore for $m \in \boldsymbol{N}^{*}, A_{r+m}^{\prime}$ only depends on $A_{r}, \ldots, A_{r+m}$.

(ii) If $A_{r+m} \in \operatorname{sl}(n, C)$ then $A_{r+m}^{\prime}$ has the same property.

Proof. The first assertion follows from Lemma 1 by taking $\mathcal{L}=\mathcal{G}_{X}$. The second one is immediate. 
To continue the reduction we may suppose according to the lemma that $A$ satisfies $A_{r+m} \in \mathcal{G}_{X}$ for $1 \leq m<\Lambda(q-r)$. Then we can write

$$
\begin{aligned}
& A_{r+m}=\sum_{j=1}^{\ell} a_{r+m, j} Z_{j}, \quad 1 \leq m<\Lambda(q-r) \\
& A_{r+m}=\sum_{j=1}^{n^{2}} a_{r+m, j} Z_{j}, \quad m \geq \Lambda(q-r) .
\end{aligned}
$$

Define

$$
E=\left\{\frac{m}{\frac{\lambda_{j}}{2}+1} \mid 1 \leq m<\Lambda(q-r), 1 \leq j \leq \ell, a_{r+m, j} \neq 0\right\}
$$

Let

$$
\beta= \begin{cases}\inf E & \text { if } E \neq \emptyset \\ \infty & \text { otherwise }\end{cases}
$$

$\alpha=\min \{q-r, \beta\}$ and $S=x^{\alpha H /(2 q)}$. It is clear that $\beta>0, \alpha>0$. According to (6) we have,

$$
\begin{aligned}
S[Y] & =\left(1+x^{-1}\right)^{\alpha H /(2 q)} x^{-\alpha / q} Y \\
S\left[Z_{j}\right] & =\left(1+x^{-1}\right)^{\alpha H /(2 q)} x^{\alpha \lambda_{j} /(2 q)} Z_{j}, 1 \leq j \leq n^{2}
\end{aligned}
$$

and

$$
\begin{aligned}
A^{\prime} \stackrel{\text { def }}{=} & S[A]=\left(1+x^{-1}\right)^{\alpha H /(2 q)}\left[I+x^{-(r+\alpha) / q}(Y+\right. \\
& \sum_{\substack{1 \leq m<\Lambda(q-r) \\
1 \leq j \leq \ell}} \frac{a_{r+m, j} Z_{j}}{x^{\frac{1}{q}\left[m-\left(\frac{\lambda_{j}}{2}+1\right) \alpha\right]}}+\sum_{\substack{m \geq \Lambda(q-r) \\
1 \leq j \leq n^{2}}} \frac{a_{r+m, j} Z_{j}}{\left.\left.x^{\frac{1}{q}\left[m-\left(\frac{\lambda_{j}}{2}+1\right) \alpha\right]}\right)\right] .}
\end{aligned}
$$

Proposition 4. Let notations be as above.

(1) If $\alpha=q-r$, then $A^{\prime}$ is of level 0 ;

(2) if $0<\alpha<q-r$, write $\alpha=\frac{r^{\prime}}{q^{\prime}}$ then

$$
\begin{gathered}
A^{\prime}=I+x^{-\left(q^{\prime} r+r^{\prime}\right) /\left(q q^{\prime}\right)}\left[Y^{\prime}+O\left(x^{-1 /\left(2 q q^{\prime}\right)}\right)\right] \in \operatorname{GL}\left(n, \mathcal{O}_{2 q q^{\prime}}\right) \\
\text { with } Y^{\prime}=Y+\sum_{(m, j) \in \Omega} a_{r+m, j} Z_{j} \text { where } \\
\Omega=\left\{(m, j) \mid 1 \leq m<\Lambda(q-r), 1 \leq j \leq \ell, a_{r+m, j} \neq 0, \alpha=\frac{m}{\frac{\lambda_{j}}{2}+1}\right\} .
\end{gathered}
$$

Moreover $Y^{\prime} \neq Y$ and

- either $Y^{\prime}$ is not nilpotent 
- or $Y^{\prime}$ is nilpotent but $d\left(Y^{\prime}\right)>d(Y)$.

Proof. (1) If $\alpha=q-r$ we have for all $m \geq \Lambda(q-r), 1 \leq j \leq n^{2}$,

$$
m-\left(\lambda_{j} / 2+1\right) \alpha \geq 0 .
$$

The new matrix $A^{\prime}$ is of level 0 .

(2) If $\alpha=\beta<q-r$, we have for all $m \geq \Lambda(q-r), 1 \leq j \leq n^{2}$,

$$
m-\left(\lambda_{j} / 2+1\right) \alpha>0 .
$$

Then $Y^{\prime}$ is in the form of the proposition and $Y^{\prime} \neq Y$. If $Y^{\prime}$ is nilpotent then, by Lemma $3, d\left(Y^{\prime}\right)>d(Y)$.

Corollary 2. Assume that $\operatorname{tr}\left(A_{r+m}\right)=0$ for $0 \leq m<\Lambda(q-r)$. If $\alpha<q-r$ and $Y^{\prime}$ is not nilpotent then it has at least two distinct eigenvalues.

Proof. The assertion follows immediately from the fact that $a_{r+m, 1}=0$ for $1 \leq m<\Lambda(q-r)$.

\subsection{Reduction of systems of level $\leq 1$.}

In this section we shall prove the following proposition that is the second part of the Theorem 1 concerning systems of level $\leq 1$.

Proposition 5. Let $q \in \boldsymbol{N}^{*}$ and $A \in \mathrm{GL}\left(n, K_{q}\right)$ be of level $\leq 1$. Then there exists $p \in q \boldsymbol{N}^{*}$ such that $A$ is equivalent under $\operatorname{GL}\left(n, K_{p}\right)$ to a canonical matrix of the form

$$
B=I+\frac{D_{1}}{x^{r_{1}}}+\cdots+\frac{D_{k}}{x^{r_{k}}}+\frac{C}{x} .
$$

Recall that the $r_{1}, \ldots, r_{k} \in \frac{1}{p} \boldsymbol{N}^{*}, r_{1}<\cdots<r_{k}<1 ; D_{1}, \ldots, D_{k} \in \operatorname{gl}(n, C)$ are diagonal, $C \in \operatorname{gl}(n, C)$ commutes with the $D_{j}(1 \leq j \leq k)$.

We begin by stating some facts which will be used in the proof.

Lemma 3. Let

$$
A=I+\sum_{m=1}^{\infty} \frac{A_{m}}{x^{m / q}} \in \mathrm{GL}\left(n, K_{q}\right) .
$$

Then we can find sequences $\left(\omega_{m}\right)_{m \geq 1}$ in $\boldsymbol{C}$ and $\left(A_{m}^{\prime}\right)_{m \geq 1}$ in $\operatorname{sl}(n, \boldsymbol{C})$ such that

$$
A=\left[\prod_{m=1}^{\infty}\left(1+\frac{\omega_{m}}{x^{m / q}}\right)\right]\left(I+\sum_{m=1}^{\infty} \frac{A_{m}^{\prime}}{x^{m / q}}\right)
$$

moreover $\omega_{m}$ and $A_{m}^{\prime}$ only depend on $A_{1}, \ldots, A_{m}$. 
Proof. For $j \in N^{*}$ define $\omega_{j} \in \mathbf{C}$ and $A^{(j)}=I+\sum_{m=1}^{\infty} \frac{A_{m}^{(j)}}{x^{m / q}}$ by

$$
\begin{array}{ll}
\omega_{1}=\frac{\operatorname{tr}\left(A_{1}\right)}{n}, & A^{(1)}=\left(1+\frac{\omega_{1}}{x^{1 / q}}\right)^{-1} A \\
\omega_{j}=\frac{\operatorname{tr}\left(A_{j}^{(j-1)}\right)}{n}, & A^{(j)}=\left(1+\frac{\omega_{j}}{x^{j / q}}\right)^{-1} A^{(j-1)} .
\end{array}
$$

We have $\operatorname{tr}\left(A_{j}^{(j)}\right)=0$ and

$$
A=\left[\prod_{j=1}^{\infty}\left(1+\frac{\omega_{j}}{x^{j / q}}\right)\right]\left(I+\sum_{j=1}^{\infty} \frac{A_{j}^{(j)}}{x^{j / q}}\right) .
$$

Lemma 4. Let $B \in \mathrm{GL}\left(n, K_{p}\right)$ be in the form

$$
B=\left(1+\frac{a_{1}}{x^{1 / p}}+\cdots+\frac{a_{p-1}}{x^{(p-1) / p}}\right) I+\frac{C}{x}+\tilde{B}
$$

where $a_{i} \in C, C \in \operatorname{gl}(n, C), \tilde{B} \in \operatorname{gl}\left(n, \mathcal{O}_{p}\right)$ with $\operatorname{ord}(\tilde{B})>1$. Then $B$ is equivalent to $\left(1+\frac{a_{1}}{x^{1 / p}}+\cdots+\frac{a_{p-1}}{x^{(p-1) / p}}\right) I+\frac{C^{\prime}}{x}$ for some $C^{\prime} \in \operatorname{gl}(n, C)$.

The proof is the same as for the Proposition 1.

Corollary 3. Let $B \in \mathrm{GL}\left(n, K_{p}\right)$ be in the form

$$
B=I+\frac{D_{1}}{x^{1 / p}}+\cdots+\frac{D_{p-1}}{x^{(p-1) / p}}+\frac{C}{x}+\tilde{B}
$$

where the $D_{j} \in \operatorname{gl}(n, C)$ are diagonal matrices, $C \in \operatorname{gl}(n, C), \tilde{B} \in \operatorname{gl}\left(n, \mathcal{O}_{p}\right)$ with $\operatorname{ord}(\tilde{B})>1, C$ and $\tilde{B}$ commute with the $D_{j}$. Then $B$ is equivalent to $I+\frac{D_{1}}{x^{1 / p}}+\cdots+\frac{D_{p-1}}{x^{(p-1) / p}}+\frac{C^{\prime}}{x}$ for some $C^{\prime} \in \operatorname{gl}(n, C)$.

Proof of the Proposition. Write

$$
A=I+\sum_{m=0}^{\infty} \frac{A_{r+m}}{x^{(r+m) / q}}, A_{r} \neq 0,1 \leq r<q .
$$

Let $\kappa(A)=\max (0, q-r)$. We will prove the proposition by induction on $(\operatorname{rank}(A), \kappa(A))$ with lexicographical order.

If $\operatorname{rank}(A)=1$, i.e. $A_{r+m} \in \mathbf{C}$ one can easily find $f \in K_{q} \backslash\{0\}$ such that

$$
A=\left(1+\frac{A_{r}}{x^{1 / q}}+\cdots+\frac{A_{r+q}}{x}\right) \frac{\phi f}{f} .
$$


If $\kappa(A)=0$ then the matrix $A$ is of level 0 (see Proposition 1 ).

Let $(\operatorname{rank}(A), \kappa(A))>(1,0)$. Assume that the proposition is true for all matrices $A^{\prime}$ such that $\left(\operatorname{rank}\left(A^{\prime}\right), \kappa\left(A^{\prime}\right)\right)<(\operatorname{rank}(A), \kappa(A))$. We distinguish two cases:

(i) $A_{r}$ has at least two distinct eigenvalues. Then by the corollary of the Lemma 1 , there exists $T \in \mathrm{GL}\left(n, \mathcal{O}_{q}\right)$ such that $T[A]=\operatorname{diag}\left(A^{\prime}, A^{\prime \prime}\right)$ where $A^{\prime} \in \mathrm{GL}\left(n^{\prime}, K_{q}\right)$ and $A^{\prime \prime} \in \mathrm{GL}\left(n^{\prime \prime}, K_{q}\right)$ are of level $\leq 1$ and $n^{\prime}, n^{\prime \prime}<n$. Then we can apply the induction to $A^{\prime}$ and $A^{\prime \prime}$.

(ii) $A_{r}$ has only one eigenvalue $\omega$.

- If $A_{r}=\omega I$, one can write $A=\left(1+\omega x^{-r / q}\right) A^{\prime}$ where

$$
A^{\prime}=I+\sum_{m=0}^{\infty} \frac{A_{r^{\prime}+m}^{\prime}}{x^{\left(r^{\prime}+m\right) / q}}, A_{r^{\prime}}^{\prime} \neq 0
$$

with $r^{\prime}>r$. One first applies the induction to $A^{\prime}$ and afterwards uses the Corollary 3 to reduce $A$.

- If $A_{r} \neq \omega I$, by using the Lemma 3 followed by the Corollary 3, one can assume that $A_{r+m} \in \operatorname{sl}(n, \mathbf{C})$ for $m \in \mathbf{N}$. Now $A_{r}$ is nonzero and nilpotent. Let $\left(A_{r}, H, X\right)$ be a standard triple. By Lemma 2 one can also suppose that $A_{r+m} \in \mathcal{G}_{X} \cap \operatorname{sl}(n, \mathbf{C})$ for $0 \leq m<\Lambda(q-r)$. Thus the Proposition 4 is applicable: There exists a shearing transformation $S$ such that $A^{\prime}=S[A]$ satisfies one of the following statements

- either $A^{\prime}$ is of level 0

- or $A^{\prime}$ is of level $\leq 1$, i.e. $A^{\prime}=I+\frac{Y^{\prime}}{x^{r^{\prime} / q^{\prime}}}+\cdots$ with $1 \leq r^{\prime}<q^{\prime}$, $q^{\prime} \in q \mathbf{N}^{*}, Y^{\prime} \in \operatorname{sl}(n, \mathbf{C}), Y^{\prime} \neq 0$ and $Y^{\prime} \neq Y$.

In the latter case:

- Either $Y^{\prime}$ is not nilpotent; hence it has at least two distinct eigenvalues since $\operatorname{tr}\left(Y^{\prime}\right)=0$. We are back in the case (i).

- or $Y^{\prime}$ is nilpotent then $d\left(Y^{\prime}\right)>d(Y)$. By repeating the same procedure a finite number of times we find in the end either the "non nilpotent" case or the "level 0" one.

\section{Reduction of general systems.}

Analogous reduction as in the preceding section reduces completely differential systems. In the difference case we need to apply one more reduction procedure to reduce general difference systems to systems of level $\leq 1$. This phenomena is unique to difference systems. We now study the reduction of general difference systems. 


\subsection{The nilpotent case.}

Let

$$
A=x^{-r / q} \sum_{m=0}^{\infty} \frac{A_{r+m}}{x^{m / q}} \in \mathrm{GL}\left(n, K_{q}\right), A_{r} \neq 0 .
$$

Assume that $Y=A_{r}$ is nilpotent. Define $\nu=q \cdot \operatorname{ord}\left(x^{n r / q} \operatorname{det} A\right)$. Then $\nu>0$.

We can begin the reduction of $A$.

(a) Let $(Y, H, X)$ be a standard triple. There exists $T \in \operatorname{GL}\left(n, \mathbf{C}\left[\frac{1}{x^{1 / q}}\right]\right)$ such that

$$
A^{\prime} \stackrel{\text { def }}{=} T[A]=x^{-r / q}\left(Y+\frac{A_{r+1}^{\prime}}{x^{1 / q}}+\cdots+\frac{A_{r+\nu \Lambda}^{\prime}}{x^{(\nu \Lambda) / q}}+\cdots\right)
$$

with $A_{r+m}^{\prime} \in \mathcal{G}_{X}$ for $m=1, \ldots, \nu \Lambda$. Furthermore for $m \in \mathbf{N}^{*}, A_{r+m}^{\prime}$ only depends on $A_{r}, \ldots, A_{r+m}$.

(b) According to (a) we may assume that $A$ already satisfies $A_{r+m} \in \mathcal{G}_{X}$ for $m=1, \ldots, \nu \Lambda$. Write

$$
\begin{aligned}
& A_{r+m}=\sum_{j=1}^{\ell} a_{r+m, j} Z_{j}, \quad 1 \leq m \leq \nu \Lambda, \\
& A_{r+m}=\sum_{j=1}^{n^{2}} a_{r+m, j} Z_{j}, \quad m>\nu \Lambda .
\end{aligned}
$$

Define

$$
E=\left\{\frac{m}{\frac{\lambda_{j}}{2}+1} \mid 1 \leq m \leq \nu \Lambda, 1 \leq j \leq \ell, a_{r+m, j} \neq 0\right\} .
$$

We claim that $E \neq \emptyset$ and $\inf E \leq \nu$. Indeed since $\operatorname{det}\left(\sum_{m=0}^{\nu} \frac{A_{r+m}}{x^{m / q}}\right) \neq 0$ one concludes that $A_{r+m} \neq 0$ for some $1 \leq m \leq \nu$.

Let $\beta=\inf E>0$ and $S=x^{\beta H /(2 q)}$. Then

$$
\begin{aligned}
S[Y] & =\left(1+x^{-1}\right)^{\beta H /(2 q)} x^{-\beta / q} Y \\
S\left[Z_{j}\right] & =\left(1+x^{-1}\right)^{\beta H /(2 q)} x^{\beta \lambda_{j} /(2 q)} Z_{j}, \quad 1 \leq j \leq n^{2}
\end{aligned}
$$

and

$$
\begin{gathered}
S[A]=\left(1+x^{-1}\right)^{\beta H /(2 q)} x^{-(r+\beta) / q}\left[Y+\sum_{\substack{1 \leq m \leq \nu \Lambda \\
1 \leq j \leq \ell}} \frac{a_{r+m, j} Z_{j}}{x^{\frac{1}{q}\left[m-\left(\frac{\lambda_{j}}{2}+1\right) \beta\right]}}+\right. \\
\sum_{\substack{m>\nu \Lambda \\
1 \leq j \leq n^{2}}} \frac{a_{r+m, j} Z_{j}}{\left.x^{\frac{1}{q}\left[m-\left(\frac{\lambda_{j}}{2}+1\right) \beta\right]}\right] .}
\end{gathered}
$$


Let $A^{\prime}=S[A]$. Write $\beta=\frac{r^{\prime}}{q^{\prime}}$ with $r^{\prime}, q^{\prime} \in \mathbf{N}^{*}$. Recall that $0<\beta \leq \nu$. For all $m>\nu \Lambda, m-\left(\frac{\lambda_{j}}{2}+1\right) \beta>0$. Then $A^{\prime} \in \mathrm{GL}\left(n, \mathcal{O}_{2 q q^{\prime}}\right)$. More precisely

$$
A^{\prime}=x^{-\frac{q^{\prime} r+r^{\prime}}{q q^{\prime}}}\left[Y^{\prime}+O\left(\frac{1}{x^{1 /\left(2 q q^{\prime}\right)}}\right)\right]
$$

where

$$
Y^{\prime}=Y+\sum_{(m, j) \in \Omega} a_{r+m, j} Z_{j}
$$

The summation is over the (non empty) set

$$
\Omega=\left\{(m, j) \mid 1 \leq m \leq \nu \Lambda, 1 \leq j \leq \ell, a_{r+m, j} \neq 0, \beta=\frac{m}{\frac{\lambda_{j}}{2}+1}\right\} .
$$

We have proved the following

Proposition 6. Let notations be as above. Then $Y^{\prime} \neq Y$ and $Y^{\prime}$ is

- either not nilpotent,

- or nilpotent, in which case $d\left(Y^{\prime}\right)>d(Y)$.

\subsection{Proof of the Theorem 1 .}

Write $A=x^{-r / q} \sum_{m=0}^{\infty} \frac{A_{r+m}}{x^{m / q}}$ with $A_{r} \neq 0$. We proceed by induction on $n$. It is clear for $n=1$. Suppose $n>1$. We assume the assertion in dimension $<n$. We distinguish two cases:

(i) $A_{r}$ is not nilpotent.

- $A_{r}$ has at least two distinct eigenvalues. Then by the Corollary 1 of the Lemma 1, there exists a matrix $T \in \mathrm{GL}\left(n, \mathcal{O}_{q}\right)$ such that $T[A]=\operatorname{diag}\left(A^{\prime}, A^{\prime \prime}\right)$ where $A^{\prime} \in \mathrm{GL}\left(n^{\prime}, K_{q}\right)$ and $A^{\prime \prime} \in \mathrm{GL}\left(n^{\prime \prime}, K_{q}\right)$ with $n^{\prime}, n^{\prime \prime}<n$. Then we apply the induction to reduce $A^{\prime}$ and $A^{\prime \prime}$.

- $A_{r}$ has only one eigenvalue $\lambda \neq 0$.

(a) $A_{r}=\lambda I$. Write $A=x^{-r / q} \lambda A^{\prime}$ where $A^{\prime}$ is a matrix of level $\leq 1$. Then one can apply the Proposition 5 .

(b) $A_{r}=\lambda I+Y, Y$ is nonzero and nilpotent. Let $(Y, H, X)$ be a standard triple. Define $S=x^{\frac{H}{2 n q}}$. Then

$$
S[A]=x^{-(n r+1) /(n q)} \lambda\left(I+\sum_{m=1}^{\infty} \frac{A_{r+m}^{\prime}}{x^{m /(2 n q)}}\right)
$$

where $A_{r+m}^{\prime} \in \operatorname{gl}(n, \mathbf{C})$. We are back to the case (a). 
(ii) $A_{r}$ is nilpotent. Let $\left(A_{r}, H, X\right)$ be a standard triple. There is no loss of generality to assume that $A_{r+m} \in \mathcal{G}_{X}$ for $1 \leq m \leq \nu \Lambda$. Then the Proposition 6 implies that there exists a shearing transformation $S=x^{\frac{r^{\prime} H}{2 q q^{\prime}}}$ such that

$$
A^{\prime}=S[A]=x^{-\frac{q^{\prime} r+r^{\prime}}{q q^{\prime}}}\left[Y^{\prime}+O\left(\frac{1}{x^{1 /\left(2 q q^{\prime}\right)}}\right)\right]
$$

Moreover

- either $Y^{\prime}$ is not nilpotent. Therefore $A^{\prime}$ is in one of the forms treated in the case (i).

- or $Y^{\prime}$ is nilpotent but $Y^{\prime} \neq Y$ and $d\left(Y^{\prime}\right)>d(Y)$. By repeating the same argument a finite number of times the "non nilpotent" case occurs. This terminates the proof.

\section{Uniqueness of the canonical form.}

Definition 2. Let $p \in \mathbf{N}^{*}$. A matrix $C \in \operatorname{gl}(n, \mathbf{C})$ is said to be $p$-reduced if $0 \leq \operatorname{Re}(\lambda)<\frac{1}{p}$ for all eigenvalues $\lambda$ of $C$.

A canonical matrix $B$ as in the Definition 1 is said to be $p$-reduced if the $C_{\alpha}^{(i)}$ are $p$-reduced.

Lemma 5. Any canonical matrix in $\mathrm{GL}\left(n, K_{p}\right)$ is equivalent to a p-reduced one.

Proof. It is sufficient to consider the case of a canonical matrix of the form $B=(1+a) I+C x^{-1}$ where $a \in \mathbf{C}\left[x^{-1 / p}\right]$ of degree $<p$ without constant coefficient. One can assume that $C$ has only one eigenvalue $\lambda$. Write $\lambda=$ $\tilde{\lambda}+\frac{r}{p}$ with $r \in \mathbf{Z}$ and $0 \leq \operatorname{Re}(\tilde{\lambda})<\frac{1}{p}$. Let $T=x^{-r / q} I$, then

$$
T\left[(1+a) I+C x^{-1}\right]=(1+a) I+(C-(r / p) I) x^{-1}+O\left(x^{-(p+1) / p}\right) .
$$

Furthermore there exists a matrix $T^{\prime} \in \operatorname{GL}\left(n, \mathcal{O}_{p}\right)$ such that

$$
T^{\prime} T\left[(1+a) I+C x^{-1}\right]=(1+a) I+(C-(r / p) I) x^{-1} .
$$

It is obvious that the latter matrix is $p$-reduced.

Theorem 2. Let $p \in \boldsymbol{N}^{*}$. Let $B=\frac{1}{x^{r / p}} \bigoplus_{i=1}^{s} \frac{B_{i}}{x^{\ell_{i}}}, B^{\prime}=\frac{1}{x^{r / p}} \bigoplus_{i=1}^{s^{\prime}} \frac{B_{i}^{\prime}}{x^{\ell_{i}^{\prime}}}$ be two canonical matrices in $\operatorname{GL}\left(n, K_{p}\right)$, where

$$
B_{i}=\bigoplus_{\alpha=1}^{t_{i}} \lambda_{\alpha}^{(i)}\left(B_{\alpha}^{(i)}+\frac{C_{\alpha}^{(i)}}{x}\right), \quad B_{i}^{\prime}=\bigoplus_{\alpha=1}^{t_{i}^{\prime}} \lambda_{\alpha}^{\prime(i)}\left(B_{\alpha}^{\prime(i)}+\frac{C_{\alpha}^{\prime(i)}}{x}\right)
$$


are as in the Definition 1, with

$$
\begin{aligned}
B_{\alpha}^{(i)} & =I_{\alpha}^{(i)}+\frac{D_{\alpha, 1}^{(i)}}{x^{r_{\alpha, 1}^{(i)}}}+\cdots+\frac{D_{\alpha, j_{\alpha}^{(i)}}^{(i)}}{x^{r^{(i)}(i)}} \in \operatorname{GL}\left(n_{\alpha}^{(i)}, \mathcal{O}_{p}\right) \\
B_{\alpha}^{\prime(i)} & =I_{\alpha}^{\prime(i)}+\frac{D_{\alpha, 1}^{\prime(i)}}{x^{r_{\alpha, 1}^{\prime(i)}}}+\cdots+\frac{D_{\alpha, j_{\alpha}^{\prime(i)}}^{\prime(i)}}{x^{r^{\prime(i)} j_{\alpha}^{\prime(i)}}} \in \operatorname{GL}\left(n_{\alpha}^{\prime(i)}, \mathcal{O}_{p}\right)
\end{aligned}
$$

which verify the conditions of the Definition 1 . Then $B$ and $B^{\prime}$ are equivalent in $\mathrm{GL}\left(n, K_{p}\right)$ iff the following conditions are satisfied

(i) $s^{\prime}=s$, for all $1 \leq i \leq s, n^{\prime(i)}=n^{(i)}, \ell_{i}^{\prime}=\ell_{i}, t_{i}^{\prime}=t_{i}$, for all $1 \leq \alpha \leq t_{i}$, $n_{\alpha}^{\prime(i)}=n_{\alpha}^{(i)}$,

(ii) for all $1 \leq i \leq s, j_{\alpha}^{(i)}=j_{\alpha}^{(i)}$ for all $1 \leq \alpha \leq t_{i}, r_{\alpha, j}^{(i)}=r_{\alpha, j}^{(i)}\left(1 \leq j \leq j_{\alpha}^{(i)}\right)$,

(iii) there exist matrices $E_{i} \in \mathrm{GL}\left(n^{(i)}, C\right)$ such that

$$
\begin{aligned}
& B_{i}^{\prime} \equiv E_{i} B_{i} E_{i}^{-1}\left(\bmod x^{-1}\right), \\
& \exp \left(2 i \pi p \oplus_{\alpha=1}^{t_{i}} C_{\alpha}^{\prime(i)}\right)=E_{i} \exp \left(2 i \pi p \oplus_{\alpha=1}^{t_{i}} C_{\alpha}^{(i)}\right) E_{i}^{-1} .
\end{aligned}
$$

Moreover if $B$ and $B^{\prime}$ are assumed p-reduced, then the condition (7) is equivalent to $B_{i}^{\prime}=E_{i} B_{i} E_{i}^{-1}$.

Proof. There is no loss of generality in assuming $r=0$. We are going to prove the theorem under the assumption that $B$ and $B^{\prime}$ are $p$-reduced. The general case can be deduced from the special one with the aid of the Lemma 5. The sufficiency is trivial. We now prove the necessity.

(a) We show at first that $\ell_{1}^{\prime}=\ell_{1}$. Let $T \in \operatorname{GL}\left(n, K_{p}\right)$ be such that $B^{\prime}=T[B]$. Write $T$ in the Smith normal form $T=P x^{-D} Q$ where $P, Q \in \operatorname{GL}\left(n, \mathcal{O}_{p}\right)$ with $\operatorname{det} P(\infty) \neq 0, \operatorname{det} Q(\infty) \neq 0 . \quad D=\operatorname{diag}\left(\alpha_{1}, \ldots, \alpha_{n}\right)$ is a diagonal matrix with $\alpha_{i} \in \frac{1}{p} \mathbf{Z}$ and $\alpha_{1} \leq \alpha_{2} \leq \cdots \leq \alpha_{n}$. Let $\hat{B}^{\prime}=P \phi(P)^{-1} B^{\prime}$ and $\hat{B}=(1+1 / x)^{-D} \phi(Q) B Q^{-1}$. One can then write

$$
\begin{gathered}
\hat{B}^{\prime}=x^{-\ell_{1}^{\prime}}\left(A_{0}^{\prime}+\cdots\right), A_{0}^{\prime}=\operatorname{diag}\left(\lambda_{1}^{\prime(1)} I_{1}^{\prime(1)}, \ldots, \lambda_{t_{1}^{\prime}}^{(1)} I_{t_{1}^{\prime}}^{\prime(1)}, 0\right) \\
\hat{B}=x^{-\ell_{1}}\left(Q_{0} A_{0} Q_{0}^{-1}+\cdots\right), A_{0}=\operatorname{diag}\left(\lambda_{1}^{(1)} I_{1}^{(1)}, \ldots, \lambda_{t_{1}}^{(1)} I_{t_{1}}^{(1)}, 0\right) .
\end{gathered}
$$

Then $x^{-D} \hat{B}(x) x^{D}=P^{-1} \hat{B}^{\prime} P$. If $\lambda$ denotes an indeterminate then one has $\operatorname{det}\left(\lambda I-x^{\ell_{1}} \hat{B}\right)=\operatorname{det}\left(\lambda I-x^{\ell_{1}} \hat{B}^{\prime}\right)$. If $\ell_{1} \neq \ell_{1}^{\prime}$ for example $\ell_{1}^{\prime}>\ell_{1}$ one would have $\operatorname{ord}\left(x^{\ell_{1}} \hat{B}\right)=0$ and $\operatorname{ord}\left(x^{\ell_{1}} \hat{B}^{\prime}\right)=\ell_{1}^{\prime}-\ell_{1}>0$ and then $\operatorname{det}\left(\lambda I-A_{0}\right)=$ $\operatorname{det}(\lambda I)=\lambda^{n}$. This contradicts to the fact that $\lambda_{1}^{(1)} \neq 0$. Hence $\ell_{1}^{\prime}=\ell_{1}$. As a consequence we have $\operatorname{det}\left(\lambda I-A_{0}\right)=\operatorname{det}\left(\lambda I-A_{0}^{\prime}\right)$. From the structure of $A_{0}^{\prime}$ and $A_{0}$ we deduce that there exits a permutation matrix $E_{0}$ such that 
$A_{0}^{\prime}=E_{0} A_{0} E_{0}^{-1}$. To simplify the notations, by considering $E_{0} B_{1} E_{0}^{-1}$ in the place of $B_{1}$, we can assume that $A_{0}^{\prime}=A_{0}$. Then $n^{(1)}=n^{(1)}, t_{1}^{\prime}=t_{1}$ and for all $\alpha=1, \ldots, t_{1}, n_{\alpha}^{(1)}=n_{\alpha}^{(1)}, \lambda_{\alpha}^{\prime(1)}=\lambda_{\alpha}^{(1)}$.

(b) Let $\tilde{B}=\oplus_{\alpha=2}^{s} x^{-\ell_{\alpha}} B_{\alpha}, \tilde{B}^{\prime}=\oplus_{\alpha=2}^{s^{\prime}} x^{-\ell_{\alpha}^{\prime}} B_{\alpha}$. Write

$$
T=\left(\begin{array}{ll}
T_{1} & T_{3} \\
T_{2} & T_{4}
\end{array}\right)
$$

where $T_{1}$ is an $n^{(1)} \times n^{(1)}$ square matrix. Then $B^{\prime} T=\phi(T) B$ becomes

$$
\begin{aligned}
& x^{-\ell_{1}} B_{1}^{\prime} T_{1}=x^{-\ell_{1}} \phi\left(T_{1}\right) B_{1}, \quad \tilde{B}^{\prime} T_{4}=\phi\left(T_{4}\right) \tilde{B}, \\
& \tilde{B}^{\prime} T_{2}=x^{-\ell_{1}} \phi\left(T_{2}\right) B_{1}, \quad x^{-\ell_{1}} B_{1}^{\prime} T_{3}=\phi\left(T_{3}\right) \tilde{B} .
\end{aligned}
$$

We claim that $T_{2}=0$. Otherwise ord $\left(T_{2}\right)<+\infty$, then

$$
\begin{gathered}
\operatorname{ord}\left(\tilde{B}^{\prime} T_{2}\right) \geq \operatorname{ord}\left(\tilde{B}^{\prime}\right)+\operatorname{ord}\left(T_{2}\right)=\ell_{2}^{\prime}+\operatorname{ord}\left(T_{2}\right), \\
\operatorname{ord}\left(x^{-\ell_{1}} \phi\left(T_{2}\right) B_{1}\right)=\ell_{1}+\operatorname{ord}\left(T_{2}\right)+\operatorname{ord}\left(B_{1}\right)=\ell_{1}+\operatorname{ord}\left(T_{2}\right) .
\end{gathered}
$$

This contradicts the fact that $\ell_{1}<\ell_{2}^{\prime}$. Then $T_{2}=0$. For the same reason one has $T_{3}=0$. Therefore $T=\operatorname{diag}\left(T_{1}, T_{4}\right)$ with $T_{1} \in \mathrm{GL}\left(n^{(1)}, K_{p}\right), T_{4} \in$ $\mathrm{GL}\left(n-n^{(1)}, K_{p}\right)$ and $B_{1}^{\prime}, \tilde{B}^{\prime}$ are equivalent respectively to $B_{1}, \tilde{B}$.

The same arguments applied to $B, B^{\prime}$ and similar computations applied to $B_{1}^{\prime}, B_{1}$ prove (i).

(c) Now it remains the case

$$
\begin{aligned}
B & =I+\frac{D_{1}}{r_{1}}+\cdots+\frac{D_{j}}{x^{r_{j}}}+\frac{C}{x} \\
B^{\prime} & =I+\frac{D_{1}^{\prime}}{r_{1}^{\prime}}+\cdots+\frac{D_{j^{\prime}}^{\prime}}{x^{r_{j^{\prime}}^{\prime}}}+\frac{C^{\prime}}{x} .
\end{aligned}
$$

By a change of basis one can also suppose that $B=\oplus_{l=1}^{t} B_{l}, B^{\prime}=\oplus_{k=1}^{t^{\prime}} B_{k}^{\prime}$ where

$$
\begin{gathered}
B_{l}=\left(1+a_{l}\right) I_{n_{l}}+\frac{C_{l}}{x} \in \mathrm{GL}\left(n_{l}, K_{p}\right), a_{l}=\frac{a_{l, 1}}{x^{r_{1}}}+\cdots+\frac{a_{l, j}}{x^{r_{j}}}, \sum_{l=1}^{t} n_{l}=n ; \\
B_{k}^{\prime}=\left(1+a_{k}^{\prime}\right) I_{n_{k}^{\prime}}+\frac{C_{k}^{\prime}}{x} \in \mathrm{GL}\left(n_{k}^{\prime}, K_{p}\right), a_{k}^{\prime}=\frac{a_{k, 1}^{\prime}}{x^{r_{1}^{\prime}}}+\cdots+\frac{a_{k, j^{\prime}}^{\prime}}{x^{r_{j^{\prime}}^{\prime}}}, \sum_{k=1}^{t^{\prime}} n_{k}^{\prime}=n ;
\end{gathered}
$$

with $C_{l}, C_{k}^{\prime} p$-reduced. Let

$$
T=\left(\begin{array}{ccc}
T_{11} & \cdots & T_{1 t} \\
\cdots & \cdots & \cdots \\
T_{t^{\prime} 1} & \cdots & T_{t^{\prime} t}
\end{array}\right)
$$


where $T_{k l}$ is an $n_{k}^{\prime} \times n_{l}$ matrix. We have

$$
\phi\left(T_{k l}\right)=B_{k}^{\prime} T_{k l} B_{l}^{-1}
$$

We have to show the following: $t^{\prime}=t$; there exists a permutation $\alpha$ of $\{1, \ldots, t\}$ such that $n_{k}^{\prime}=n_{\alpha(k)} ; T_{k, \alpha(k)} \in \mathrm{GL}\left(n_{k}^{\prime}, K_{p}\right), T_{k, s}=0$ for $s \neq \alpha(k)$; $a_{k}^{\prime}=a_{\alpha(k)} ; C_{k}^{\prime}$ and $C_{\alpha(k)}^{\prime}$ are similar.

Fix $1 \leq k \leq t^{\prime}$, then since $T$ is invertible there exists at least $1 \leq l \leq t$ such that $T_{k l} \neq 0$. Let $u$ be a nonzero coefficient of $T_{k l}$ with minimal order. It follows from (8) that $u$ satisfies an equation of the following form

$$
\frac{\phi(u)}{u}=\frac{1+a_{k}^{\prime}}{1+a_{l}}+\frac{f}{x}
$$

with $f \in K_{p}$ and $\operatorname{ord}(f) \geq 0$. Since $\frac{\phi(u)}{u} \in 1+\frac{1}{x} \mathcal{O}_{p}$ one deduces that $a_{k}^{\prime}=a_{l}$.

Since $a_{k} \neq a_{s}$ for $k \neq s$ one has $T_{k, s}=0$. Write $l=\alpha(k)$ the unique index such that $T_{k l} \neq 0$. This shows that $t^{\prime}=t$ and $\alpha$ is a permutation of $\{1, \ldots, t\}$. Furthermore we have $n_{k}^{\prime}=\operatorname{rank}\left(T_{k, \alpha(k)}\right)=n_{\alpha(k)}$ and then $T_{k, \alpha(k)}$ is invertible. Now (8) implies immediately that $C_{k}^{\prime}, C_{\alpha(k)}$ are similar. This completes the proof.

The authors thank the referee for helpful comments and suggestions.

\section{References}

[1] D.G. Babbitt and V.S. Varadarajan, Formal reduction theory of meromorphic differential equations: A group theoretic view, Pacific J. Math., 109 (1983), 1-80.

[2] G. Chen, Sur la réduction formelle des systèmes aux différences, C. R. Acad. Sci. Paris, Série I, 322 (1996), 1145-1150.

[3] _ Forme normale d'Arnold et réduction formelle de systèmes d'équations linéaires aux différences, Aequationes Math., to appear.

[4] A. Duval, Lemme de Hessel et factorisation formelle pour les opérateurs aux différences, Funkcial Ekvac., 26 (1983), 349-368.

[5] G. Immink, Asymptotics of analytic difference equations, Springer L. N. in Math., 1085 (1984).

[6] B. Kostant, The principal three-dimensional subgroup and the betti numbers of a complex simple Lie group, Amer. J. Math., 81 (1959), 973-1032.

[7] C. Praagman, The formal classification of linear difference operators, Proc. Kon. Ned. Ac. Wet. Ser A, 86 (1983), 249-261. 
[8] H.L. Turrittin, The formal theory of systems of irregular homogeneous linear difference and differential equations, Bol. Soc. Math. Mexicana, 5 (1960), 255-264.

Received March 15, 1996 and revised February 11, 1997.

URA CNRS 751

UNIVERSITÉ DE LILLE 1

59655 Villeneuve D'AscQ, France

E-mail address: Guoting.Chen@univ-lille1.fr AND

UNIVERSITÉ D'ANGERS

49045 ANGERs, France

E-mail address: fahim@tonton.univ-angers.fr 